\title{
Photoacoustic tomography with a virtual point detector
}

Xinmai Yang, Meng-Lin Li, Lihong V. Wang

Xinmai Yang, Meng-Lin Li, Lihong V. Wang, "Photoacoustic tomography with a virtual point detector," Proc. SPIE 6437, Photons Plus Ultrasound: Imaging and Sensing 2007: The Eighth Conference on Biomedical Thermoacoustics, Optoacoustics, and Acousto-optics, 643718 (13 February 2007); doi: 10.1117/12.698367

SPIE. Event: SPIE BiOS, 2007, San Jose, California, United States 


\title{
Photoacoustic tomography with a virtual point detector
}

\author{
Xinmai Yang, Meng-Lin Li, and Lihong V. Wang \\ Optical Imaging Laboratory, Department of Biomedical Engineering, Washington University in St. \\ Louis, St. Louis, Missouri 63130
}

\begin{abstract}
We devise and explore a ring-shaped acoustic detector associated with a virtual point detector concept for photoacoustic tomography. The center of the ring transducer scans a circle around the object to be imaged and then is treated as an omni-directional virtual point detector in photoacoustic image reconstruction. The virtual point detector introduces a space-invariant point spread function in photoacoustic image reconstruction and thus improves the tangential resolution, which is due to the finite aperture. Compared with a real point detector, the virtual point detector can provide similar spatial resolution but better SNR. Compared with a real finite-aperture detector, the virtual point detector can provide similar SNR but better spatial resolution. In addition, because of its virtual feature, the virtual point detector can be placed very close to and even inside of a tissue sample to locally scan a region of interest, which yields good SNR and spatial resolution.
\end{abstract}

Keywords: photoacoustic tomography, resolution, image reconstruction, virtual point detector

\section{INTRODUCTION}

Photoacoustic tomography (PAT) has shown great potentials in the field of biomedical imaging [1][2]. The spatial resolution of PAT is limited by the laser pulse width, the bandwidth of the photoacoustic (PA) detection system, and the aperture size of the detector [3]-[8]. In practice, the first parameter is usually sufficiently short, whereas the second parameter can be easily varied to reach desired resolution at the expense of penetration. The third parameter can also be reduced to yield high spatial resolution at the cost of signal-noise ratio (SNR). However, due to large noise, a real point detector cannot be used in PAT.

In this paper, we propose, for the first time to our knowledge, a ring-based virtual point detector and demonstrate its application in PAT. The virtual point detector provides PAT with the advantages of a real point detector, i.e., high spatial resolution that is uniform throughout the field of view. At the same time, it produces much lower thermal noise than a real point detector because of its larger actual size. Below, we will first analyze the principle of the virtual point detector, then present simulation and experimental data, and finally discuss the results. Here, the study is limited to two dimensions (2D) but can be extended to three dimensions.

\section{THEORETICAL ANALYSIS}

When a PA pulse from a point PA source is received by a real point ultrasonic detector, the received pressure is $p_{p}(t)$, which is the convolution between the received signal and the impulse response of the transducer, where $t$ denotes time. When the PA pulse is received by a ring-shaped ultrasonic detector, the received pressure becomes $p_{r}(t)=\int_{0}^{2 \pi} \frac{p_{p}(t-R(\theta) / c)}{R(\theta)} d \theta$, where $\theta$ denotes the polar angle of a point on the detector surface with respect to 
a)

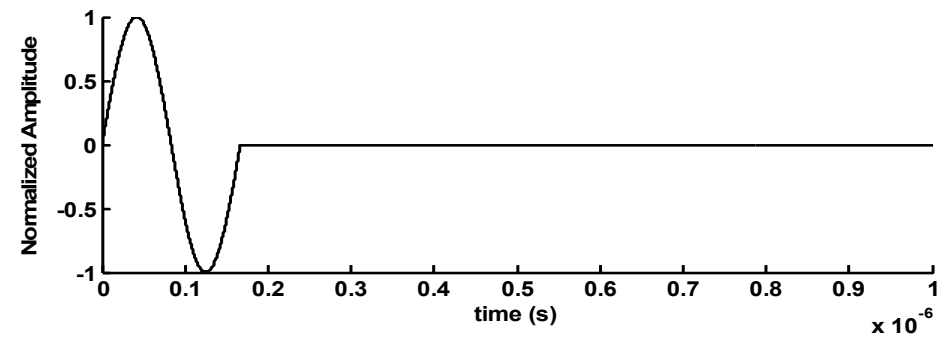

b)
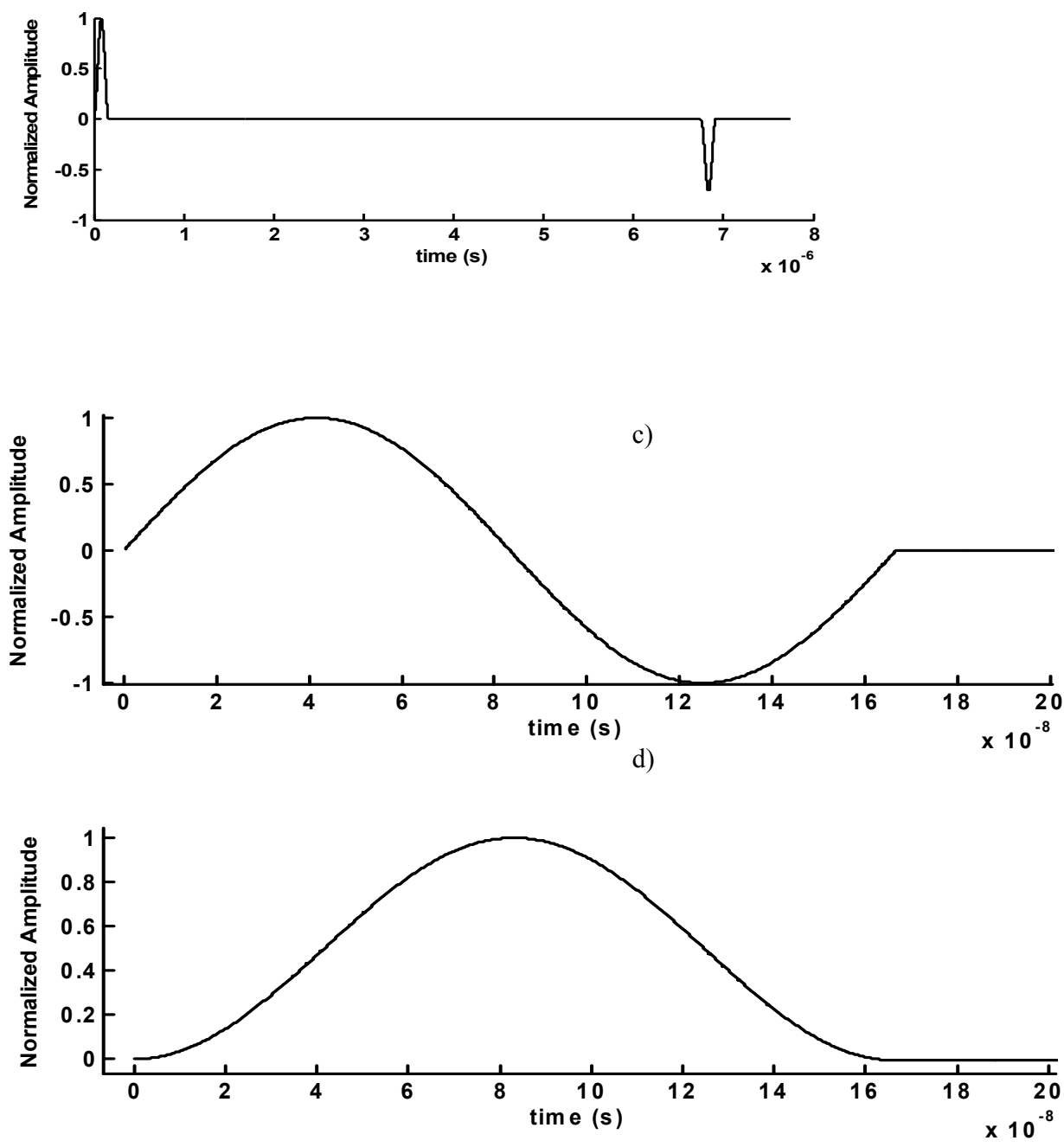

Fig. 1 In response to a one-cycle sinusoidal pulse, (a) simulated signal detected by a point detector; (b) simulated signal detected by a ring detector; and (c) enlarged pulse of the (a). (d) enlarged first pulse in (b)

the center of the ring, $R$ denotes the distance between the point source and the point on the detector surface at $\theta . c$ denotes the speed of sound in the medium. 
If we assume that $p(t)$ is a single cycle sinusoidal burst, then for a point detector, the detected signal is shown in Fig. 1(a). If the detector has a large size, e.g., has a ring shape, then the detected signal has a result as Fig. 1(b). Here we assumed the source was not at the ring center. The enlarged pulses were shown in Fig 1(c) and (d). There are three comments worthy to be made about the result: 1) the shape of the detected signal from the point detector are as same as the original signal; 2) it seems that only the first and last arrivals appear for the ring case, and notably, the two pulses have only positive or negative portion; 3) all pulses have the same length as the original pulse, which means lower frequency components are presented in the signal detected by the ring detector. Further analysis reveals that for a ring detector, the position of the first and last arrivals are symmetric according to the signal from the ring center.

From a more general point of view, a point detector and a ring detector are two extremes, whereas a finite-aperture detector lies in between. Since a point detector has a zero size, there is only one arrival on it. By contrast, a ring detector has the largest possible angular aperture, and there are two arrivals that are completely separated from each other. A small finite-aperture detector, however, has a finite size, so the arrivals (could be more than two) partially overlap. A time derivative of the output signal of a small finite-aperture detector will not easily recover the original pulse because of this overlapping. A point detector may provide the best spatial resolution. But the extremely small aperture size of a point detector yields immense noise. An alternative method is to use a ring detector to recover the original signal through its time derivative. By this mean, we can obtain a resolution similar to a real point detector but with the noise levels of a ring detector. This idea motivates us to use a ring detector.

When a ring detector is used to detect signals from a point target, the first arrival signal on the ring detector is due to the wave that propagates away from the ring center through the shortest path, which is along the radius. By contrast, the last arrival signal on a ring detector is due to the wave that propagates the longest path through the ring center. Therefore, both the first and last arrival signals can be considered to have propagated through the ring center although the first arrival signal does not physically traverse the ring center. The ring center thus can be treated as a virtual point detector, whereas the first arrival signal has a negative time delay and the last arrival signal has a positive time delay. We can separately use these two arrivals. When we separate the two arrivals on the received signal, we follow the process below,

1. identify the position of the ring-center signal on the time axis, say $t_{c}$, and divide the original signal into two signals from that position (with $t_{c}$ included in both parts);

2. perform the following calculation

$$
\begin{aligned}
& t_{f}=t_{c}-t \text { for } t \leq t_{c} \\
& t_{s}=t-t_{c} \text { for } t \geq t_{c}
\end{aligned}
$$

where $t_{f}$ and $t_{s}$ represent the new time axis for the first signal and the second signal;

After this process, either of the signals thus obtained can be used to evaluate the spatial resolution. If a circular scanning is performed, the scanning radius is calculated from the ring center to the scanning center.

While maintaining good resolution, the virtual point detector also has a low thermal noise, which in turn results in a high SNR. The thermal-noise-induced voltage can be written as $\left\langle v^{2}\right\rangle=4 k T_{0} / C_{T}$, where $C_{T}$ is the capacitance of the detector, $k$ is the Boltzmann constant, and $T_{0}$ is the temperature of the sensor. In this relation, the thermal-noiseinduced voltage is inversely proportional to the capacitance of a detector. Because the capacitance is proportional to the area of a detector, the thermal-noise-induced voltage is inversely proportional to the area. Therefore, owing to large thermal noise, using a point detector is not practical even though it has good resolution. A virtual point detector, which is constructed by a ring detector with a large surface area, however, has much lower thermal noise than a point detector.

\section{RESULTS}

We apply the virtual point detector in PAT and results are shown below. A 2D circular scanning and simple backprojection algorithm were employed. The scanning radius was $15 \mathrm{~mm}$, and the scanning center is set as the position $(0$, 
0 ) in the following figures. In our experiments, a schematic for the $2 \mathrm{D}$ scan in Fig. 2 was employed when a virtual point detector was used. As an example, Fig. 3 shows the trace of a point source in experiment detected by a virtual point detector during a circular scan. The trace of the first and the last arrivals are clearly identified on the plot, and the center of the ring, which is the line of symmetry, is drawn on the plot. For further reference, we also show the results from a finite-aperture detector. We use $30 \mathrm{~mm}$, instead of $15 \mathrm{~mm}$, as the scanning radius for the finite-aperture detector because $15-\mathrm{mm}$ is too close to the finite-aperture detector and results in very poor resolution.

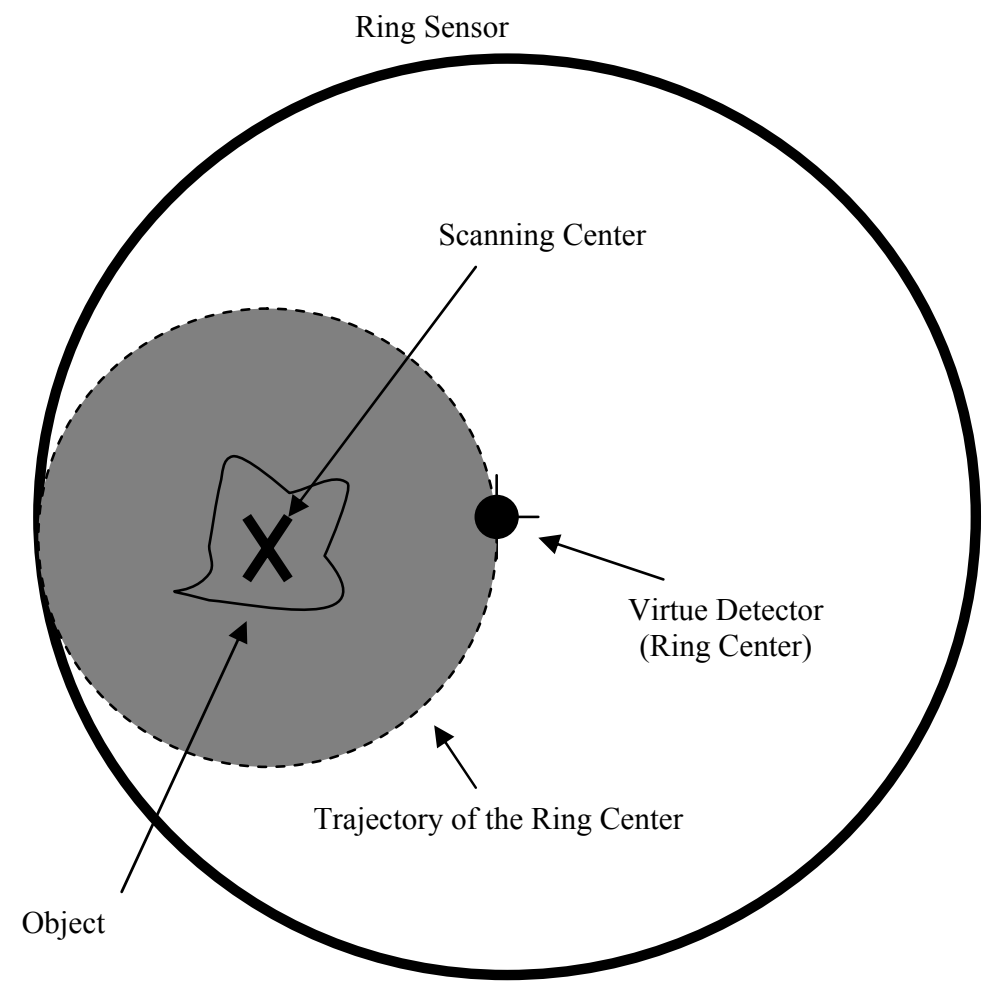

Fig. 2 Schematics for a 2D scanning by using a ring detector. 


\section{From a point source}

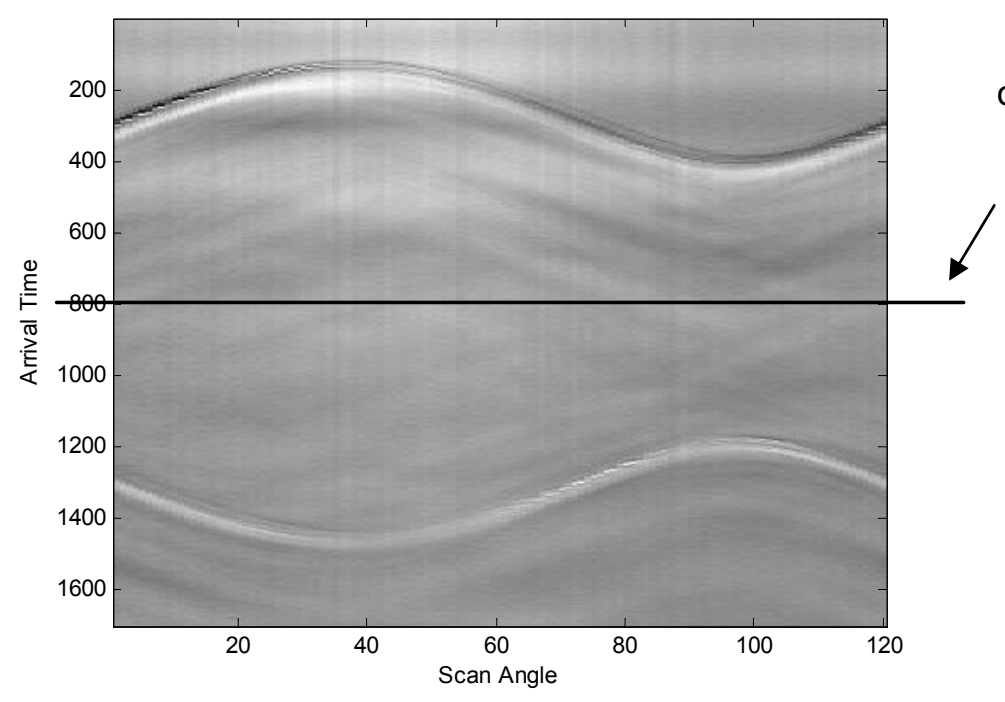

Line of Symmetry (center of the ring)

Fig. 3 Detected signals of a point source made a ring detector during a circular scan

To obtain experimental results, we built a virtual point detector with PVDF film. The thickness of the PVDF film was $110 \mu \mathrm{m}$, and the final structure had a center frequency around $6 \mathrm{MHz}$. We also need to build a real point detector. We acknowledge that a real point detector without a dimension does not exist, but we can build a near-point detector with very small size. A 4-mm-wide finite-aperture detector was also built for reference. The targets in the experiment were black threads of $20 \mu \mathrm{m}$ diameters. The diameters of these threads were one order of magnitude smaller than the wavelength at $6 \mathrm{MHz}$, and could be treated as nearly ideal point targets when they were placed vertical to the scanning plane. The laser light at the wavelength of $532 \mathrm{~nm}$ from a Q-switched Nd:YAG laser (LS-2137/2, Symphotic TII Co. Camarillo, CA), operating at the second harmonic wave, was employed as the irradiation source. The laser pulse duration was $6.5 \mathrm{~ns}$ and the pulse repetition rate is $10 \mathrm{~Hz}$. Compared with ultrasound duration (178 ns @6 MHz), the laser pulse is very short and the generated photoacoustic signal can be treated as impulses. Samples were irradiated from the top and the scan was performed on the horizontal plane. The generated ultrasound signal was detected by ultrasound detectors, and then amplified by $50 \mathrm{~dB}$ (5072 PR, Panametrics, Waltham, MA). After that, the signal was directed to a digital oscilloscope (TDS5054, Tektronix, Beaverton, OR), and then collected by a PC through a LabVIEW program.

Experimental results are shown in Fig. 4-6 Fig. In all images shown below, the scanning center is at position $(256,256)$. 4(a) and (b) show the reconstructed images of point sources from the virtual point detector, and the finite-aperture detector, respectively. In Fig. 4(a), the virtual point detector displays constant spatial resolution of $\sim 230 \mu \mathrm{m}$ in all the scanning area. At the same time, the virtual point detector maintains a relatively strong SNR. In the result shown here, the obtained SNRs are 29, 39, 29, 30, and $24 \mathrm{~dB}$. The images obtained by the finite-aperture detector have good resolutions near the scanning center, and decreasing as the targets are away from the scanning center. The SNRs obtained by the finite-aperture detector are 2436,3020 , and $15 \mathrm{~dB}$. Comparing the SNRs between these images, we find that the SNRs yielded by the virtual detector are more uniformly distributed.

Experimental results for line sources are presented in Fig 5. Fig. 5(a) shows the images obtained by a virtual point detector and Fig. 5(b) shows the images obtained by the finite-aperture detector. The advantage of the virtual point detector is clear displayed. Throughout the whole imaging region, the images of lines made by the virtual point detector display the uniform and good resolutions. But the images of lines made by the finite-aperture detector only display good resolution at the center region. Towards the ends of the lines, huge bifurcations show up and the resolutions become very poor. Although at the center region, the line images made by the finite-aperture detector show higher SNR, at the ends of these lines, the SNR becomes very poor. In Fig. 6, we show the image of a 0.6-mm diameter pencil lead inside 
a chicken breast. The thickness of the chicken breast is about $1 \mathrm{~cm}$. Again, the ring-based virtual point detector shows better resolution than that of the finite-aperture detector.

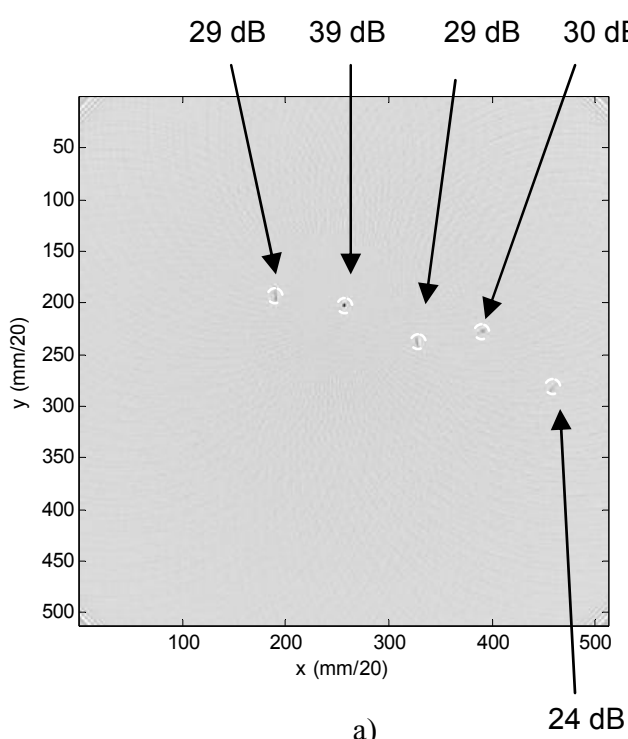

a)

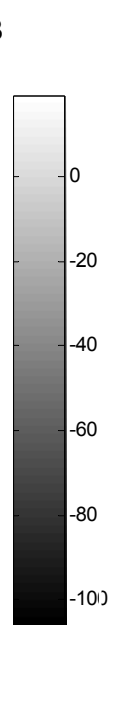

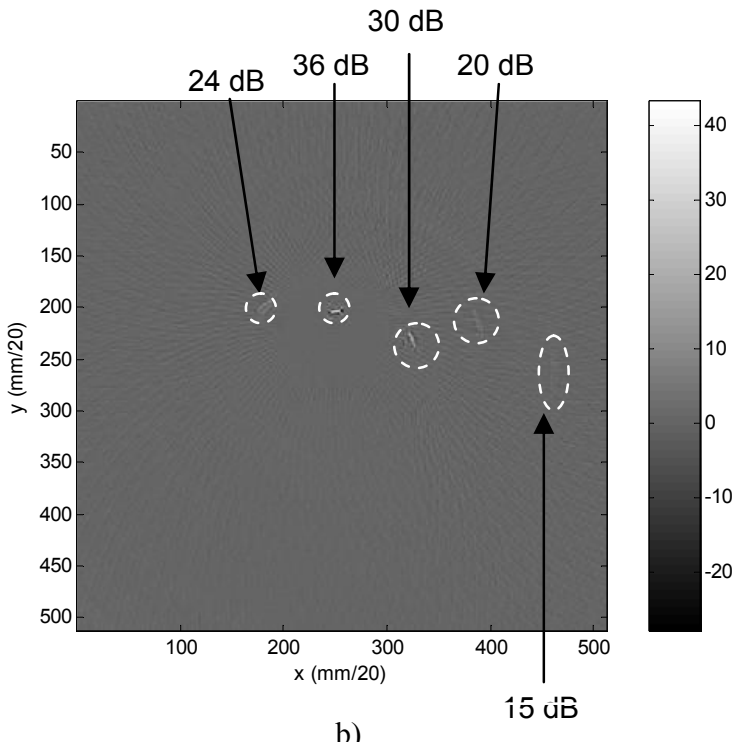

b)

Fig. 4 Images of point sources made by (a) a ring detector and (b) a finite-size-detector

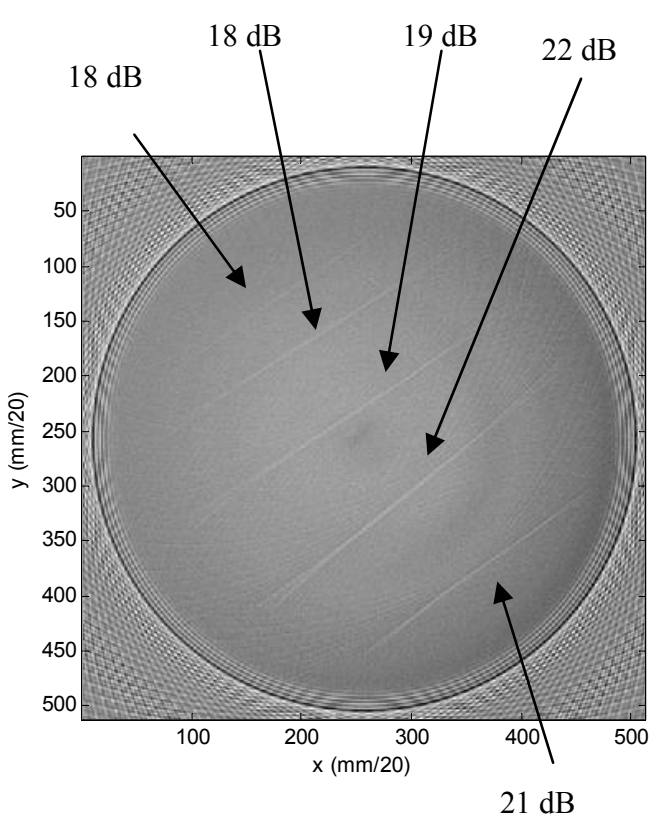

a)

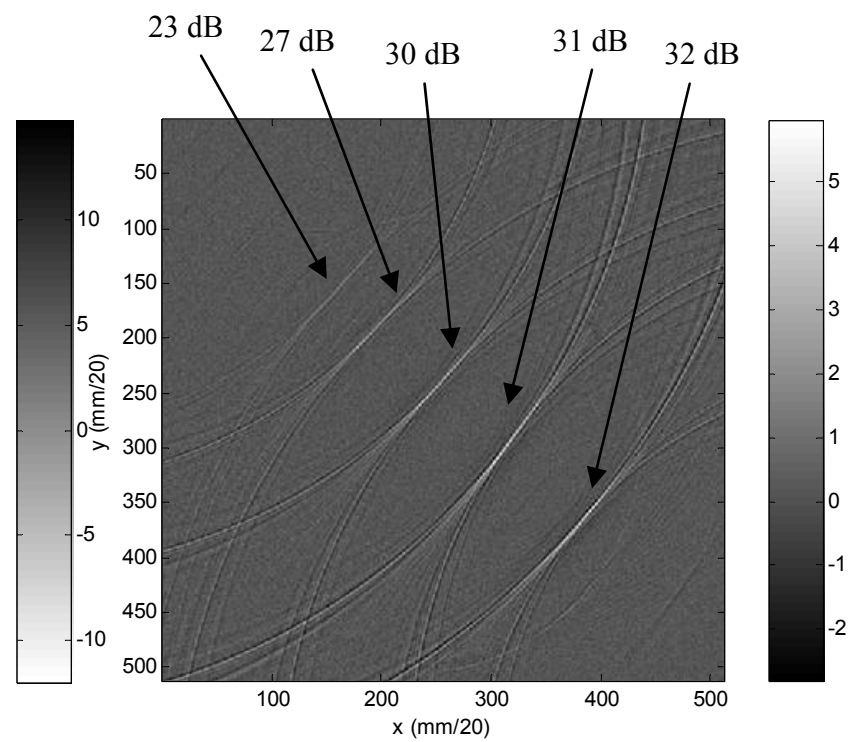

b)

Fig. 5 Images of line sources made by (a) a ring and (b) a finite-aperture detector 


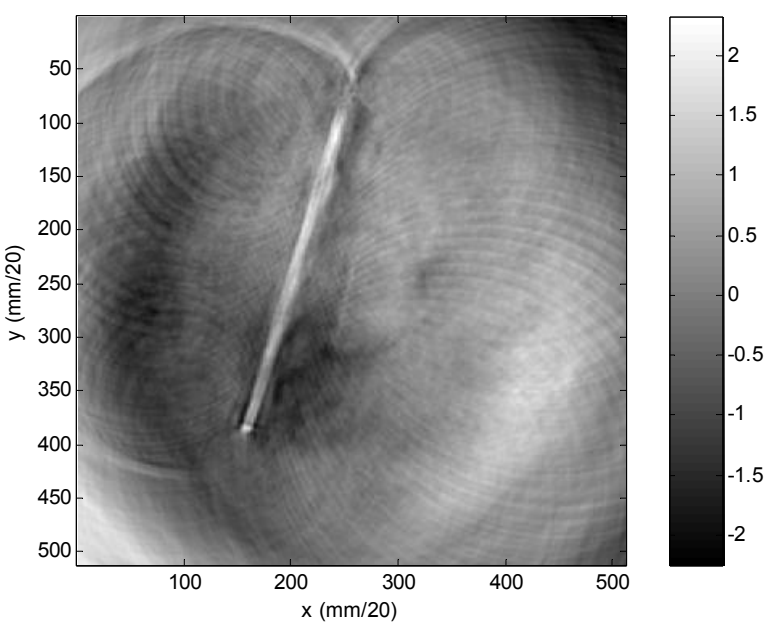

a)

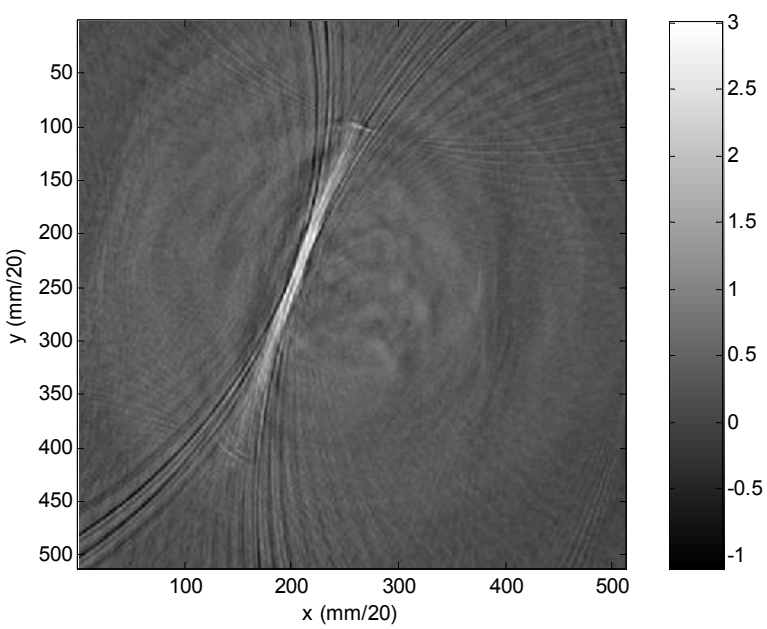

b)

Fig. 6 Image of a pencil lead in chicken breast made by (a) a ring detector and (b) a finite-aperture detector

\section{DISCUSSION}

In above images from the virtual point detector, we used the first arrival of the signal for the virtual point detector. The SNR can be further improved if the acoustic heterogeneity is taken into account, and the two arrival signals are added together and reconstructed. One more advantage of the virtual point detector is that it can be placed inside a sample and used to scan a small area to improve the local SNR. Since the virtual point detector is the focal point of the ring detector, as an object gets closer to the focal zone, the detected signal certainly will get stronger and thus yields a better SNR.

The virtual point detector yields great resolutions and a strong, more-uniformly-distributed SNR. Even when an object is outside the scanning region as defined in Fig. 2, the virtual point detector can still detect the object because the detection of the detector is omni-directional. However, the resolution may be reduced because the object is not scanned at all angles.

In conclusion, we have analyzed the resolution of photoacoustic detection. Accordingly, we have presented a virtual point ultrasonic detector and showed it has a good uniform resolution and SNR.

\section{ACKNOWLEDGEMENTS}

The authors would like to thank National Institutes of Health for the funding support through grant No. R01 CA106728 and R01 NS46214 (BRP). 


\footnotetext{
${ }^{1}$ A. A. Oraevsky and L. V. Wang, Proc. SPIE, 6086 (2006).

${ }^{2}$ M. Xu, and L. V. Wang, Rev. Sci. Instrum. 77, 041101, 1-22, (2006).

${ }^{3}$ A. A. Oraevsky and A. Karabutov, Proc. SPIE, 3619, 228-239, (2000).

${ }^{4}$ A. A. Oraevsky, R. O. Esenaliev, S. L. Jacques, S. Thomsen and F. K. Tittel, Proc. SPIE, 2389, 198-208 (1995).

${ }^{5}$ A. A. Oraevsky, R O. Esenaliev, S. L. Jacques, and F. K. Tittel, Proc. SPIE, 2676, 22-31, (1996).

${ }^{6} \mathrm{G}$. Ku and L. V. Wang, Med. Phys. 28(1), 4-10 (2001).

${ }^{7}$ M. Xu, G. Ku and L. V. Wang, Med. Phys. 28 (9), 1958-1963 (2001).

${ }^{8}$ M. Xu and L. V. Wang, Phys. Rev. E 67056605 (2003).
} 